FERMILAB-PUB-05/379-T

\title{
Observational Constraints on Undulant Cosmologies
}

\author{
Gabriela Barenboim \\ Departament de Física Teòrica, Universitat de València, Carrer Dr. Moliner 50, \\ E-46100 Burjassot (València), Spain \\ E-mail: Gabriela.Barenboim@uv.es \\ Olga Mena Requejo \\ Theoretical Physics Department, Fermi National Accelerator Laboratory, \\ P.O. Box 500, Batavia, Illinois 60510 USA \\ E-mail: omena@fnal.gov \\ Chris Quigg \\ Theoretical Physics Department, Fermi National Accelerator Laboratory, \\ P.O. Box 500, Batavia, Illinois 60510 USA \\ E-mail: quigg@fnal.gov
}

\begin{abstract}
In an undulant universe, cosmic expansion is characterized by alternating periods of acceleration and deceleration. We examine cosmologies in which the dark-energy equation of state varies periodically with the number of $e$-foldings of the scale factor of the universe, and use observations to constrain the frequency of oscillation. We find a tension between a forceful response to the cosmic coincidence problem and the standard treatment of structure formation.
\end{abstract}

Submitted to: Journal of Cosmology and Astroparticle Physics

PACS numbers: 98.80.Cq, 95.35.+d, 98.70.Vc

\section{Introduction}

Astronomical observations have led to the inference that the Universe is approximately flat, and that its complement of mass-energy now consists of $5 \%$ ordinary matter, $22 \%$ nonbaryonic dark matter, and a dominant negative-pressure component that accelerates the Hubble expansion [1, 2, 3. The discovery that the present Universe is expanding at an accelerating pace arose in measurements of distant supernova redshifts 4, 5. Detailed studies of anisotropies in the cosmic microwave background radiation [6] and broad surveys of large-scale structure [7, 8] have deepened and broadened the initial evidence. Remarkably, most of the stuff of the Universe appears to lie outside our quotidian experience, and has not yet been detected in the laboratory.

The spottiness of the fossil record that we read in distance-redshift correlations, microwave anisotropies, and large-scale structure leaves much room for interpretation [9]. The most economical description of the cosmological parameters 
attributes the negative-pressure ("dark energy") component to a cosmological constant in Einstein's equation - an omnipresent and invariable vacuum energy density that assumes a greater importance as the Universe expands [10. On this picture, we are entering a final inflationary epoch in which the Universe will grow so quickly as to be essentially empty of matter. A dynamical alternative attributes the accelerated expansion to a cosmic scalar field that changes with time and varies across space, slowly approaching its ground state [11, 12]. Such quintessence models, as they are called, admit a broad variety of future Universes.

Neither the cosmological constant interpretation nor the cosmic-scalar picture has a ready explanation for the rough balance between matter and vacuum energy at this moment in cosmic history. The cosmic coincidence problem - the "why now?" question-has stimulated speculations that range from anthropic rationalizations [13] to cyclic cosmologies [14].

Recently, we investigated the possibility that the physical characteristics of the vacuum energy might vary with time, specifically with the number of $e$-foldings of the scale factor $a$ of the Universe [15. We showed that the simple Ansatz,

$$
w_{v}(a)=-\cos (\ln a),
$$

for the vacuum-energy equation of state is compatible with existing observations and offers an intriguing response to the "why now?" problem. (We choose a phase implicit in the form (11) to match the inference that $w_{v 0} \approx-1$ in the current Universe.) The cosmic expansion of the ensuing undulant universe is characterized by alternating eras of acceleration and deceleration. Because over one period the equation of state (11) averages to zero (the equation of state of pressureless matter), the vacuum energy density tracks the matter density on average. In this way, the cosmic coincidence problem is resolved. The future evolution of the undulant universe is similar in broad outline to that of a matter-dominated universe at critical density, for which $a \propto t^{2 / 3}$, where $t$ measures the age of the Universe.

The oscillatory equation of state (11) could represent, for instance, the effective description of a dynamical cosmic field (fluid) present since very early times. In that spirit, Barenboim and Lykken [16] have extended the undulant universe notion, taking a new approach to quintessential inflation that not only yields inflation but also offers a dark energy candidate. Both features emerge from the evolution of a single scalar field in a potential with oscillatory and exponential behavior. (The potential of Ref. 16] resembles the motion of a Slinky® spring toy descending a staircase, so the paradigm is called slinky inflation.) The vacuum-energy equation of state that emerges from their potential has the form

$$
w_{v}(a)=-\cos (b \ln a),
$$

a simple generalization of Equation (11), in which the dimensionless parameter $b$ controls the frequency of inflationary epochs. They present an illustrative example with $b=\frac{1}{7}$ that reproduces measured energy densities in the present universe and produces the requisite inflation. After the end of the radiation-dominated era, indeed, back to $a \approx 10^{-8}$, the characteristics of slinky inflation are essentially identical to those of an undulant cosmology specified by the equation of state (2). At earlier times, it is necessary to solve the coupled differential equations for the radiation, matter, and vacuum-energy densities given by Equation (12) of Ref. [16.

It is interesting to observe that equations of state involving the functional form $\cos (\ln a)$ but passing through $w=-1$ have been explored, to a different end, in a number of recent papers [17, 18, 19, 20, 21]. 
In this paper we catalogue the observational tests to which we have subjected the undulant universe defined by the simple equation of state (11). We further explore the bounds that can be placed on the frequency parameter $b$ by requiring consistency with current observations. In the limit of small values of $b \rightarrow 0$, the equation of state (2) approaches the cosmological-constant value, $w \approx-1$. In that limit, the periodic equation of state merely reproduces the success of the cosmological constant + Cold Dark Matter $(\Lambda \mathrm{CDM})$ hypothesis (and offers no insight into the "why now" problem). More to the point, we find that a periodic equation of state with $0.6 \lesssim b \lesssim 2$, which responds to the cosmic coincidence problem, is in comfortable agreement with all observations, save for the demands of structure formation as expressed through linear evolution of density perturbations. We shall explore this vulnerability.

We examine ways in which future observations might further constrain, or rule out, variants of the undulant universe. The fact that an alternative so different from the $\Lambda \mathrm{CDM}$ hypothesis is compatible with observations makes it important to pursue the discovery of the accelerating expansion on multiple fronts. In addition to seeking to characterize the dark energy through its equation of state in the recent past, it is highly desirable to probe the state of the Universe at epochs for which we have not yet learned to read the fossil record. It is premature to converge on a single hypothesis.

\section{The essence of the undulant universe}

Let us recapitulate the main elements of an evolving universe. The expansion of the universe is determined by the Friedmann equation,

$$
H^{2} \equiv(\dot{R} / R)^{2}=8 \pi G_{\mathrm{N}} \rho / 3-k / R^{2}+\Lambda / 3,
$$

where $H$ is the Hubble parameter, $R$ is the cosmological scale factor, $G_{\mathrm{N}}$ is Newton's constant, $\rho$ is the energy density, $k=(+1,0,-1)$ is the curvature constant, and $\Lambda$ is a possible cosmological constant. If $\Lambda=0$, the curvature constant determines cosmic destiny. For $k=+1$ (closed Universe), the Universe recollapses in finite time; for $k=0$ (flat) and $k=-1$ (open), the Universe expands without limit. It is convenient to define the dimensionless scale factor, $a=R / R_{0}$, where the subscript 0 denotes the value at the current epoch. The critical density, defined from (3), is $\rho_{c}=3 H^{2} / 8 \pi G_{\mathrm{N}}$. The dimensionless cosmological density parameter is defined relative to the critical density as $\Omega_{\text {tot }}=\rho / \rho_{c}$ at any epoch. We express the rate of change of the Hubble parameter through the deceleration parameter,

$$
q \equiv-\frac{1}{H^{2}} \frac{\ddot{R}}{R}=\frac{\Lambda}{3 H^{2}}-\frac{4 \pi G_{\mathrm{N}}}{3 H^{2}}(\rho+3 p),
$$

where $p$ is the isotropic pressure. If we define $\Lambda=4 \pi G_{\mathrm{N}} \rho_{\Lambda}$ and introduce the equation of state $w_{i}=p_{i} / \rho_{i}$ for any component of the universe, we can recast the deceleration parameter as

$$
q=\frac{1}{2} \sum_{i} \Omega_{i}\left(1+3 w_{i}\right)=\frac{1}{2}\left(\Omega_{\mathrm{tot}}+3 \sum_{i} \Omega_{i} w_{i}\right) .
$$

The equation of state of pressureless matter is $w_{m}=0$, and that of radiation is $w_{r}=\frac{1}{3}$. We see by inspection of (4) that $w_{\Lambda}=-1$. Note that the deceleration parameter is defined to be positive if the rate of expansion is slowing; for the reference case (SCDM) of a matter-dominated universe at critical density $\left(\Omega_{\mathrm{tot}}=1, \Lambda=0\right), q=\frac{1}{2}$.

The $\Lambda$ CDM proposal is attractive for its simplicity, and it agrees well with all observations. It does appear to bear a burden of unnaturalness, however. While $\Lambda \mathrm{CDM}$ predicts that $\Omega_{\Lambda} \approx \Omega_{m}$ at some point during the evolution of the Universe, 


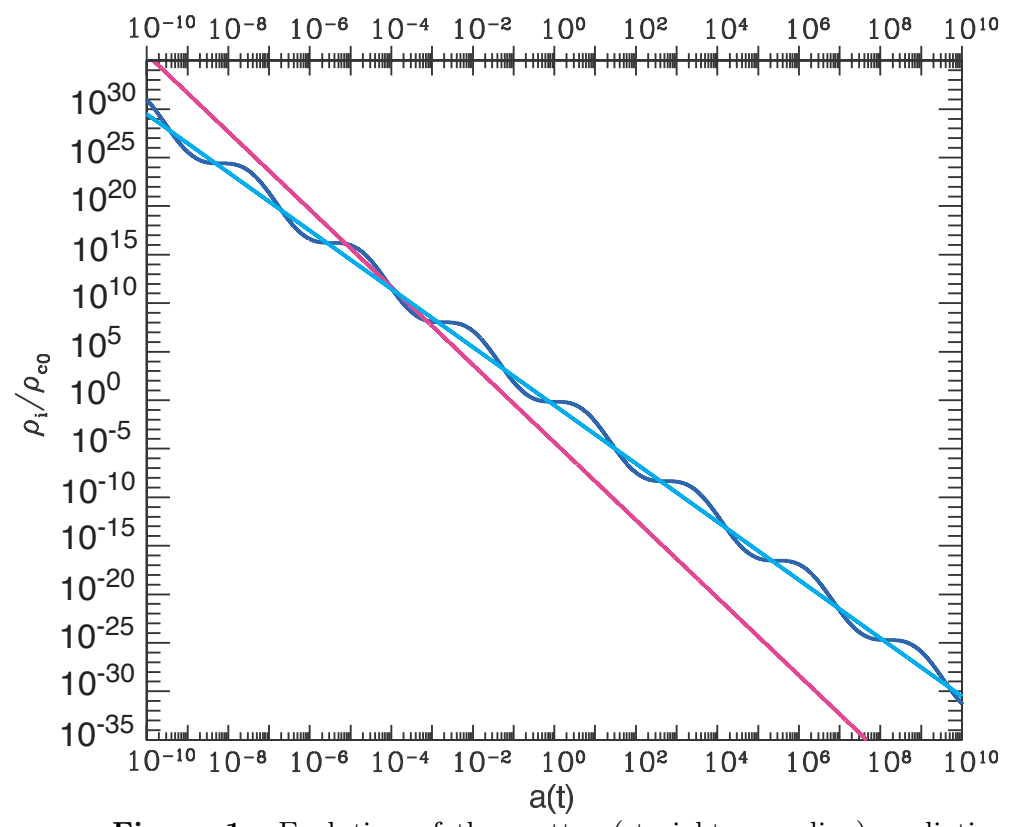

Figure 1. Evolution of the matter (straight cyan line), radiation (magenta, steepest line), and vacuum (undulating blue line) energy densities in the undulant universe, normalized to the critical density $\rho_{i} / \rho_{c 0}$, versus the scale factor $a(t)$.

it offers no explanation for the curious circumstance that the balance occurs at the current epoch - and no other - in the history of the Universe.

Let us analyze what happens if the physical characteristics of the vacuum energy vary periodically with the number of $e$-foldings of the scale factor according to the equation of state (11) or the generalization (2). In the numerical examples that follow, we assign the vacuum energy a weight $\Omega_{v 0}=0.7$, in line with observations, and take $\Omega_{m 0}=0.3$ and $\Omega_{r 0}=4.63 \times 10^{-5}$. The present-day expansion rate is $H_{0}=100 h \mathrm{~km} \mathrm{~s}^{-1} \mathrm{Mpc}^{-1}$, with $h=0.71_{-0.03}^{+0.04}$ [22].

In the general case (2), these are given in terms of the present normalized densities as $\rho_{m} / \rho_{c 0}=\Omega_{m 0} / a^{3}, \rho_{r} / \rho_{c 0}=\Omega_{r 0} / a^{4}$, and $\rho_{v} / \rho_{c 0}=g(a) \Omega_{v 0} / a^{3}$, where

$$
g(a)=e^{3 \int_{a}^{1} d a^{\prime} w\left(a^{\prime}\right) / a^{\prime}}=\exp \left[\frac{3}{b} \sin (b \ln a)\right] .
$$

We plot in Figure 1 the normalized energy densities of matter, radiation, and vacuum energy as functions of the scale parameter $a$, for the undulant universe case of $b=1$. Looking back in time to the epoch of big-bang nucleosynthesis at $a \approx 10^{-10}$, and forward to $a=10^{+10}$, we see that the vacuum energy density crosses the matter density every $\pi e$-foldings of the scale factor. If we require the vacuum component to have negative pressure, a situation similar to the present occurs every $2 \pi e$-foldings. Periodically dominant dark energy is in the spirit of Refs. 223, 24, 25, 26]. These regular crossings stand in sharp contrast to the $\Lambda$ CDM cosmology, in which $\Omega_{v} \approx \Omega_{m}$ only in the current epoch.

The presence of the exponential factor $e^{3 / b}$ in (6) raises the possibility that for small values of $b$, the excursions in the vacuum energy density about the matter density may be amplified to unacceptable - or at least highly nonstandard-levels. We plot in Figure 2 the histories of the Universe that result from the choices $b=2, \frac{2}{5}, \frac{1}{4}, \frac{1}{7}$. 

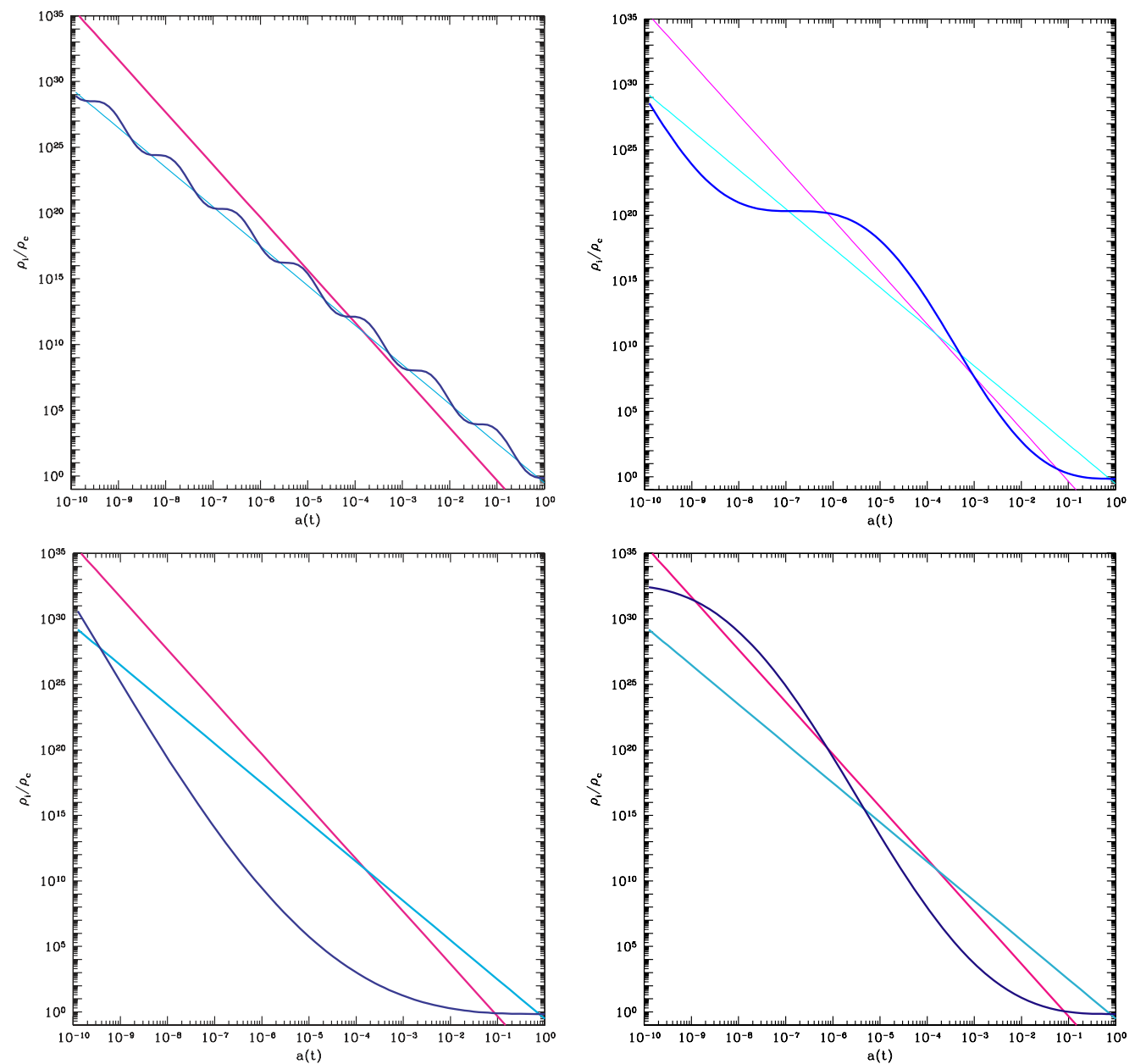

Figure 2. Evolution from $a=10^{-10}$ to the present of the matter (straight cyan line), radiation (magenta, steepest line), and vacuum (undulating blue line) energy densities in the variants of the undulant universe, normalized to the critical density $\rho_{i} / \rho_{c 0}$, for different frequency parameters $b$. Clockwise from top left, the plots correspond to $b=2, b=\frac{2}{5}, b=\frac{1}{4}$, and $b=\frac{1}{7}$.

For $b=2$ (top left panel), the oscillations are more frequent, and smaller, than those for the canonical choice $b=1$. For $b=\frac{2}{5}$ (top right), the oscillations are larger, but less frequent, than for the default undulant Universe. The radiation-dominated era ends a little earlier than in the standard cosmology, but nothing is overtly wrong. The case $b=\frac{1}{4}$ (bottom right) is highly problematic, however: the first positive excursion occurs in the era of big-bang nucleosynthesis (BBN), and would imply a large vacuumenergy component at that time. [The equation of state is $w_{v}=-0.86,-0.45,+0.11$ for $a=10^{-10}, 10^{-9}, 10^{-8}$.] At still smaller values of $b$, including the case $b=\frac{1}{7}$ (bottom left), the first positive excursion occurs well before BBN, and would not raise any obvious problems.

Although the matter density in the Universe scales smoothly as $a^{-3}$ with respect to today's critical density, the matter fraction of mass-energy at any moment, $\rho_{m}(a) / \rho_{\operatorname{tot}(a)}$, is influenced by the evolution of the rest of the portfolio. In the case 


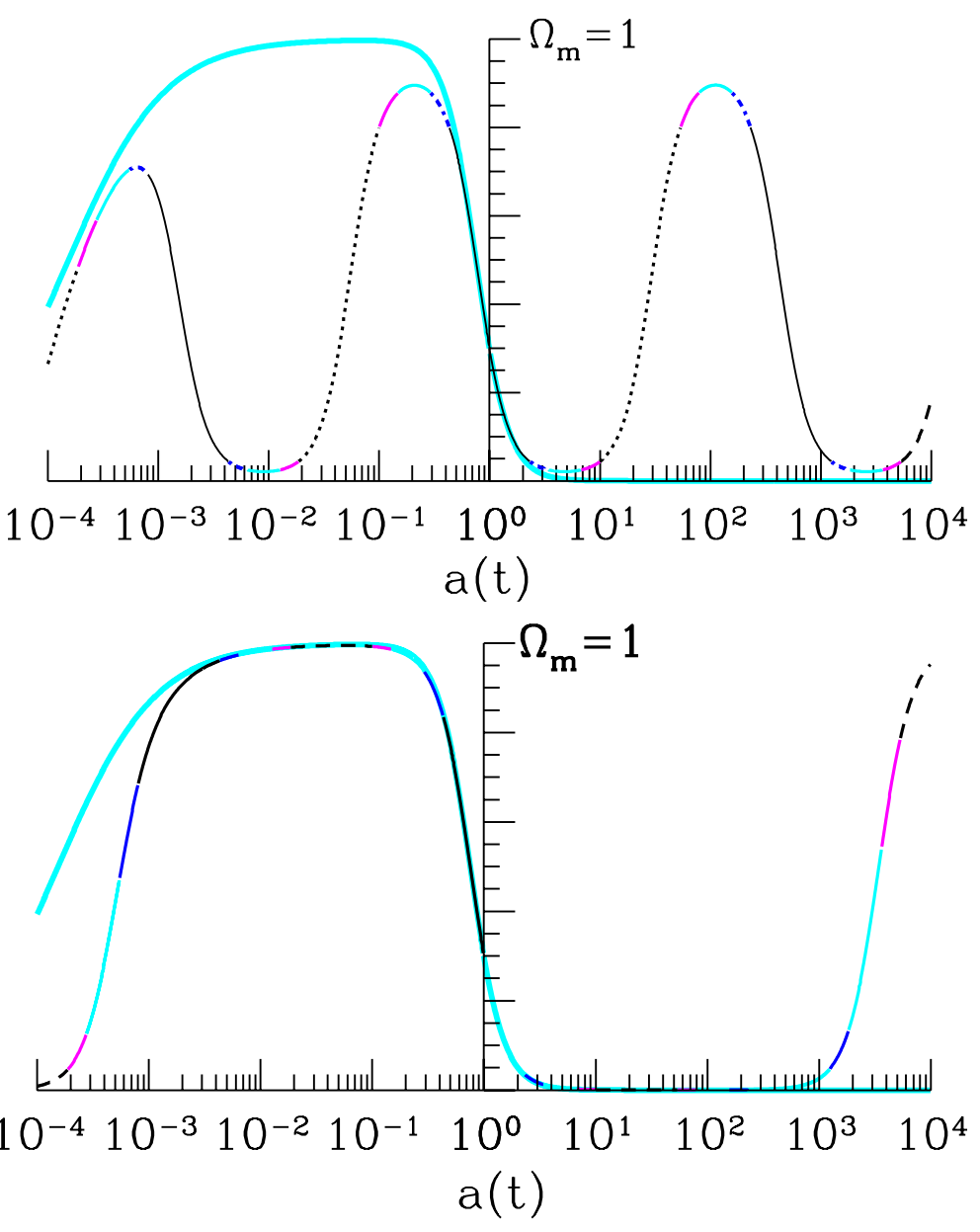

Figure 3. Evolution of the matter fraction as a function of scale factor for the $\Lambda$ CDM model (thick cyan curve) and for the undulant universe described by (1) (oscillatory curve). At different eras, the vacuum fluid emulates radiation (magenta), matter (cyan), small negative values of $w$ (blue, dash-dotted), dark energy (black solid), and a scalar field in the kination regime (dotted). Top panel: undulant universe of (1); bottom panel: generalized undulant universe of (2), with frequency parameter $b=\frac{2}{5}$.

of the $\Lambda \mathrm{CDM}$ picture, the matter density was dominant in the recent past and will be totally negligible in the near future, because the vacuum energy density due to the cosmological constant is independent of scale factor. The resulting evolution is shown by the thick curve in Figure 3 matter takes over from radiation as the dominant component as the scale factor increases through $a=10^{-4}$. At recent times, corresponding to $a \gtrsim 10^{-1}$, matter is supplanted by vacuum energy once and for all, and quickly becomes a negligible fraction of the mass-energy budget.

In the undulant cosmology implied by the equation of state (11), the matter fraction is periodically dominant, not only at the end of the radiation era, but also at regular intervals in the future. The approximate cycles repeat every $2 \pi / b e$-foldings, where $b$ is the frequency parameter in (2). The changing equation of state of the 

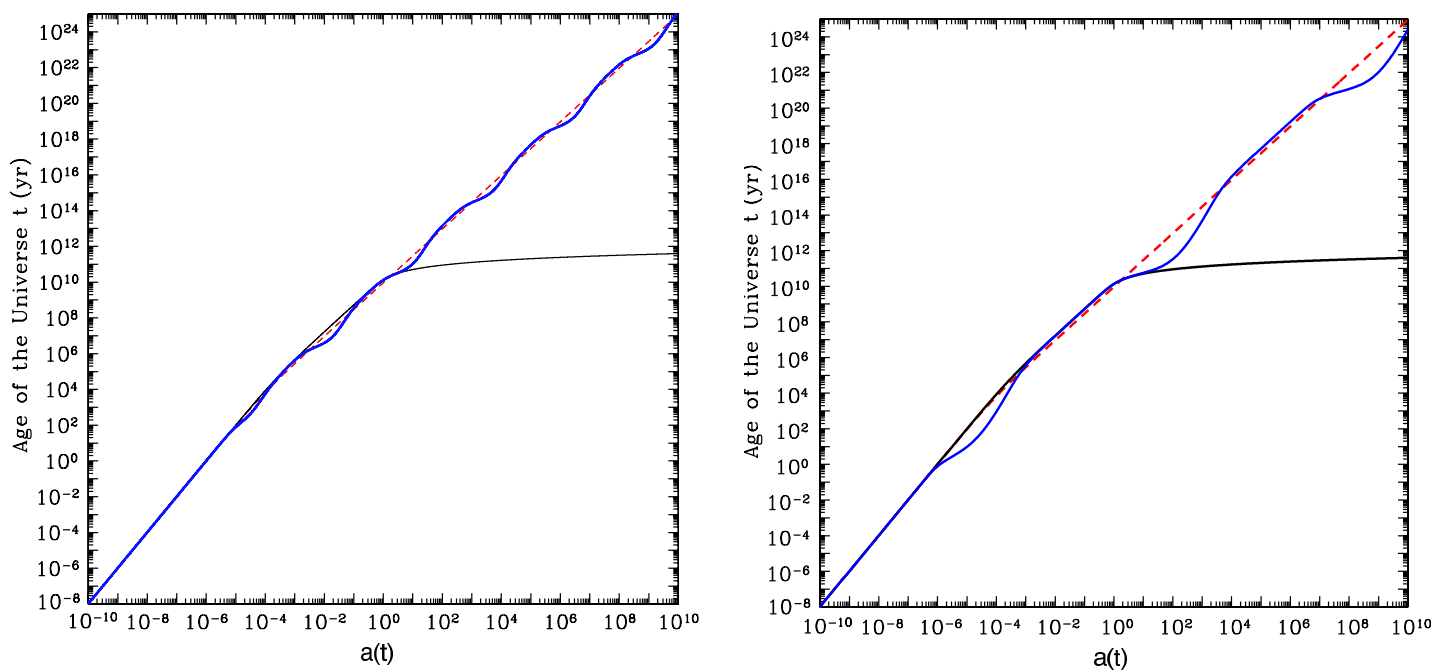

Figure 4. Evolution of the scale factor $a(t)$ in three cosmologies: the canonical $\Lambda$ CDM model (thin black line); a critical universe (SCDM model) with $\Omega_{m}=1$ (dashed red line); and (left panel) the periodic equation of state (1) or (right panel) the periodic equation of state (2) with $b=0.4$ (thick blue line).

vacuum energy is encoded in the color and texture of the undulating curve in Figure 3 The dotted black line corresponds to $1>w>\frac{2}{3}$; magenta to $\frac{2}{3}>w>\frac{1}{3}$; cyan to $\frac{1}{3}>w>-\frac{1}{3}$; dash-dotted blue to $-\frac{1}{3}>w>-\frac{2}{3}$; solid black to $-\frac{2}{3}>w>-1$.

The Hubble parameter corresponding to scale factor $a$ is given by

$$
H(a)=H_{0} \sqrt{\frac{\Omega_{m}}{a^{3}}+\frac{g(a) \Omega_{v}}{a^{3}}+\frac{\Omega_{r}}{a^{4}}} .
$$

In the undulant universe (with $b=1$ ), the current age of the universe, $t_{0}=$ $\int_{0}^{1} d a / H(a) a$, is $13.04 \mathrm{Gyr}$, to be compared with $13.46 \mathrm{Gyr}$ in the $\Lambda \mathrm{CDM}$ model. By calculating the time to reach a given scale factor, we can determine the history and future of the universe. During the radiation-dominated era, which corresponds to $a \lesssim 10^{-5}, a(t) \propto t^{1 / 2}$; when matter dominates, $a(t) \propto t^{2 / 3}$.

We show the time dependence of the scale factor $a(t)$ for three cosmologies in Figure 4 The dashed (red) line corresponds to the "standard cold dark matter" (SCDM) cosmology that was canonical before the discovery of the accelerating universe. The thin solid (black) line shows the $\Lambda \mathrm{CDM}$ cosmology, in which the present epoch marks the beginning of a final inflationary period that leads to an empty universe in which matter is a negligible component. The heavy (blue) line shows the prediction that follows from Equation (11). In the recent past, the periodic equation of state matches the behavior of the $\Lambda \mathrm{CDM}$ cosmology, but in the future it undulates about the SCDM prediction.

The alternating periods of acceleration and deceleration that characterize the expansion of undulant universes are signaled by the deceleration parameter in Figure $\mathbf{5}$, For scale factors $a$ between 0.1 and 1 , the periodic equation of state (11) tracks the behavior of the $\Lambda \mathrm{CDM}$ cosmology. For the generalized form (2), undulant cosmologies trace the $\Lambda \mathrm{CDM}$ curve over greater or smaller ranges of the scale factor for values of the frequency parameter $b$ that are smaller or greater than unity. In contrast to the 


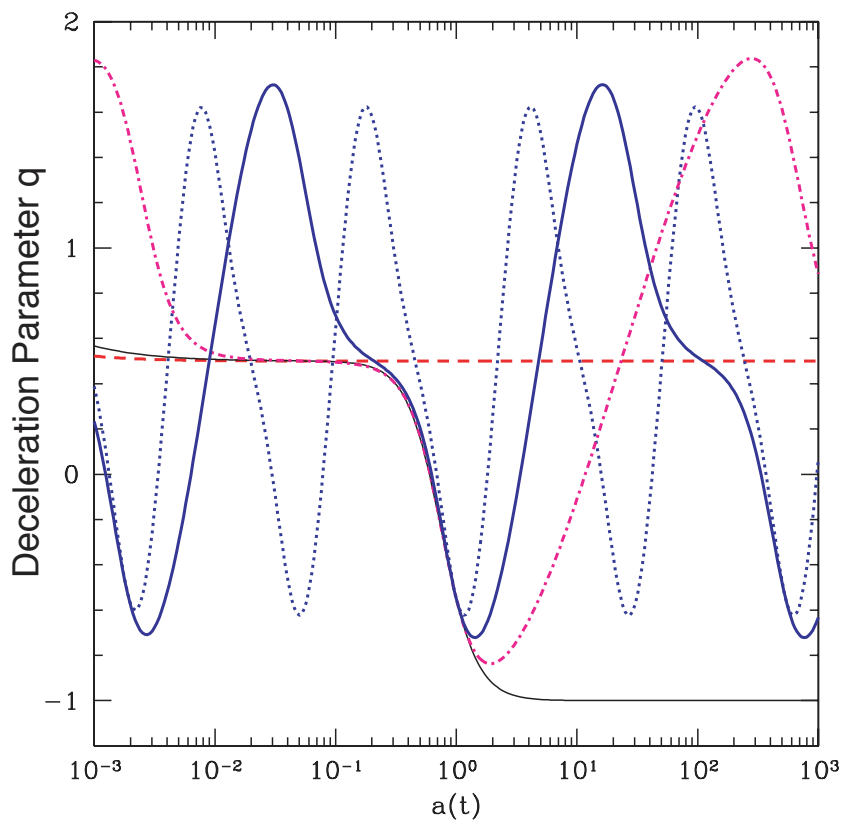

Figure 5. The deceleration parameter as defined in Equation (5) for the undulant universe of (1) (thick blue line), $\Lambda$ CDM model (thin black line) and SCDM model (dashed red line). Variants of the undulant universe given by the generalized equation of state (2) are shown as the dot-dashed line, for $b=\frac{1}{2}$, and the dotted line, for $b=2$.

$\Lambda$ CDM universe, which about to enter a final inflationary era of sustained acceleration, the average behavior of the undulant universes tracks that of SCDM.

Let us take a closer look at the "why now?" problem. In the $\Lambda$ CDM picture, there is literally no (other) time like the present, but in undulant cosmologies the matter-dark-energy coincidence is a more or less typical circumstance. We will need to define a statistical measure to assess how typical is the present state of the Universe. It is informative to distribute a large number $\mathcal{N}$ of points randomly in $\ln (a)$, excluding a slice that contains the present Universe, and to ask what fraction correspond to a balance between matter and dark energy similar to what we observe today. We define the current epoch by the condition $0.7<a<1.6$, and say that the current situation is matched, provided that

$$
\begin{aligned}
& 0.1 \leq \frac{\Omega_{m}}{\Omega_{m}+\Omega_{v}} \leq 0.5, \text { and } \\
& 0.5 \leq \frac{\Omega_{v}}{\Omega_{m}+\Omega_{v}} \leq 0.9, \text { with } w_{v}<-0.7 .
\end{aligned}
$$

[For scale factors $a \gtrsim 10^{-5}$, radiation contributes negligibly to the energy portfolio of the Universe, so that $\Omega_{m}+\Omega_{v} \approx 1$.]

We examine two intervals in the scale factor. First, we consider responses to the cosmic coincidence question since the era of radiation dominance $a>10^{-5}$, when the Universe was about 94 years old, and a corresponding step into the future, extending to $a=10^{5}$. We tally the number of successful throws of $\ln (a)$, out of $\mathcal{N}=10^{6}$ trials, and report the probability of success in Table 1 In the undulant universe defined 
Table 1. Probability, in percent, that conditions approximate the current matterdark-energy energy balance of the Universe, according to the criteria explained in the text, for various values of the frequency parameter $b$ of [2].

\begin{tabular}{|c||c|c||c|c|}
\hline$b$ & $10^{-5}<a<0.7$ & $1.6<a<10^{5}$ & $10^{-20}<a<0.7$ & $1.6<a<10^{20}$ \\
\hline $0(\Lambda \mathrm{CDM})$ & 0 & 0 & 0 & 0 \\
\hline 0.1 & 0 & 0 & 0 & 0 \\
\hline 0.125 & 0 & 0 & 0 & 0 \\
\hline $1 / 7$ & 0 & 0 & 1.5 & 1.6 \\
\hline 0.25 & 0 & 0 & 1.6 & 1.6 \\
\hline 0.4 & 0 & 0 & 3.2 & 3.2 \\
\hline 0.5 & 0 & 0 & 4.8 & 4.8 \\
\hline 0.6 & 6.4 & 6.4 & 6.4 & 6.4 \\
\hline 0.7 & 6.4 & 6.4 & 7.8 & 8.0 \\
\hline 0.9 & 6.5 & 6.5 & 9.8 & 9.8 \\
\hline 1 & 6.6 & 6.7 & 11.3 & 11.5 \\
\hline 2 & 18.2 & 18.2 & 21.1 & 21.2 \\
\hline 5 & 24.9 & 24.9 & 24.8 & 25.3 \\
\hline
\end{tabular}

by $b=1$ [15], success comes about one time in sixteen, both in the past and in the future. The current conditions are in that sense typical of the undulant universe. This statistical conclusion squares with the behavior we observed in Figure 11 As the frequency of undulations increases, conditions like those in the current Universe become commonplace. On the other hand, for values of the frequency parameter $b \lesssim 0.6$, the present coincidence of mass and dark energy is the only one to occur in the range $10^{-5} \leq a \leq 10^{5}$. For the range $10^{-7} \leq a \leq 10^{7}$, values of $b \lessgtr \frac{3}{8}$ yield only the present coincidence.

Expanding the range over we look for cosmic coincidences to the interval $10^{-20} \leq$ $a \leq 10^{20}$, we find that the undulant universe with $b=\frac{1}{7}$ implies one coincidence in the past and one in the future, but that models in which $b \lesssim \frac{1}{7}$ imply no coincidences other than the present one, which is included by design. It is fair to question whether a matter-dark-energy coincidence in the radiation-dominated universe $\left(a \lesssim 10^{-5}\right)$ is of any moment. The tracking behavior of slinky inflation, in contrast, ensures that radiation is not uniformly dominant from the big bang to $a \approx 10^{-5}$, and opens the way to early coincidences that are more generally meaningful.

\section{Tests of Undulant Cosmologies}

The principal constraints on cosmological models arise from observational knowledge of the conditions that prevailed at the time of big-bang nucleosynthesis, studies of the cosmic microwave background that look back to the surface of last scattering $\left(a \approx 10^{-3}\right)$, and measurements of the deceleration parameter in supernova redshift surveys near the present epoch, extending to redshifts $z \approx 2$. The growth of largescale structure also exhibits some sensitivity to the cosmic equation of state, through a tension between the attraction of gravitational stability and the dynamical friction of the expansion, but the standard treatment involves only linear perturbations [27]. 
We shall discuss these in turn.

\subsection{Big-bang nucleosynthesis}

The presence of a dark energy field at early times alters the expansion rate of the Universe, changing the ratio of neutrons to protons at freeze-out and modifying the predicted abundances for light elements. At those early times $\left(a \lesssim 10^{-9}\right)$ the vacuumenergy density implied by the undulant universe (10) is utterly negligible in comparison to radiation, as we saw in Figure 1 and so its influence on big-bang nucleosynthesis will be imperceptible. We find, for example, $\Omega_{v}\left(a=10^{-10}\right) \approx 2 \times 10^{-5}$, and $\Omega_{v}\left(a=10^{-9}\right) \approx 10^{-6}$.

Bean, Hansen, and Melchiorri 28] have reported that the ${ }^{4} \mathrm{He}$ mass fraction and the deuterium-to-hydrogen ratio do not favor the presence of a dark-energy component. In a class of quintessence models, they determine the bound $\Omega_{v}<0.045$ at $2 \sigma$. We can take the resulting bound as a reasonable upper limit for the more general case of the periodic equation of state (2). This bound is not threatened for $0.5 \lessgtr b \lesssim 2$. However, the exponential factor $e^{3 / b}$ in the expression (6) for $g(a)$ can grow so large, for small values of the frequency parameter $b$, that the energy budget of the Universe is altered dramatically just at the BBN era. We see this effect in the $b=1 / 4$ (bottom right) panel of Figure 2 The large value $\Omega_{v}^{b=1 / 4}\left(a=10^{-9}\right) \approx 0.4$ is clear cause for concern. Smaller values, including $b=\frac{1}{7}$, for which a large positive excursion of the vacuum-energy density occurs well before $\mathrm{BBN}$, do not provoke any concern on this score.

\subsection{Power spectrum}

To explore the implications of the periodic equation of state for anisotropies of the cosmic microwave background, we have made the appropriate modifications to the CMBFAST [29] code, assuming three massless neutrinos and fixing the helium mass fraction at $y_{\mathrm{He}}=0.24$. We show in Figure 6 that the undulant universe describes the angular power spectrum, temperature-polarization cross-correlation, and matter power spectrum with the same degree of fidelity as the $\Lambda \mathrm{CDM}$ model. The structure at large scales $(\ell \lesssim 10)$ in the $T-E$ cross correlation is a consequence of reionization [33]. Similar agreement can be expected for $b \lesssim 2$ : small values of the frequency parameter resemble the $\Lambda \mathrm{CDM}$ picture, and values close to $b=1$ yield similar averages of the vacuum-energy equation of state.

\subsection{SN Ia luminosity distance}

Type Ia supernovae now constitute an incisive probe of the state of the Universe near the present epoch. Existing data provide good resolution in the redshift range $0 \lesssim z \lesssim 1.7$. In the concordance $(\Lambda \mathrm{CDM})$ model inferred from these and other recent data, this is the range in which dark energy works its influence on the cosmic expansion rate.

The observational technique consists in determining the apparent magnitude $m$ (essentially the logarithm of the observed flux) and the redshift $z$. The apparent magnitude is related to the absolute magnitude $M$ of the supernova through the luminosity distance

$$
d_{\mathrm{L}}=c(1+z) \int_{0}^{z} \frac{d z^{\prime}}{H\left(z^{\prime}\right)},
$$



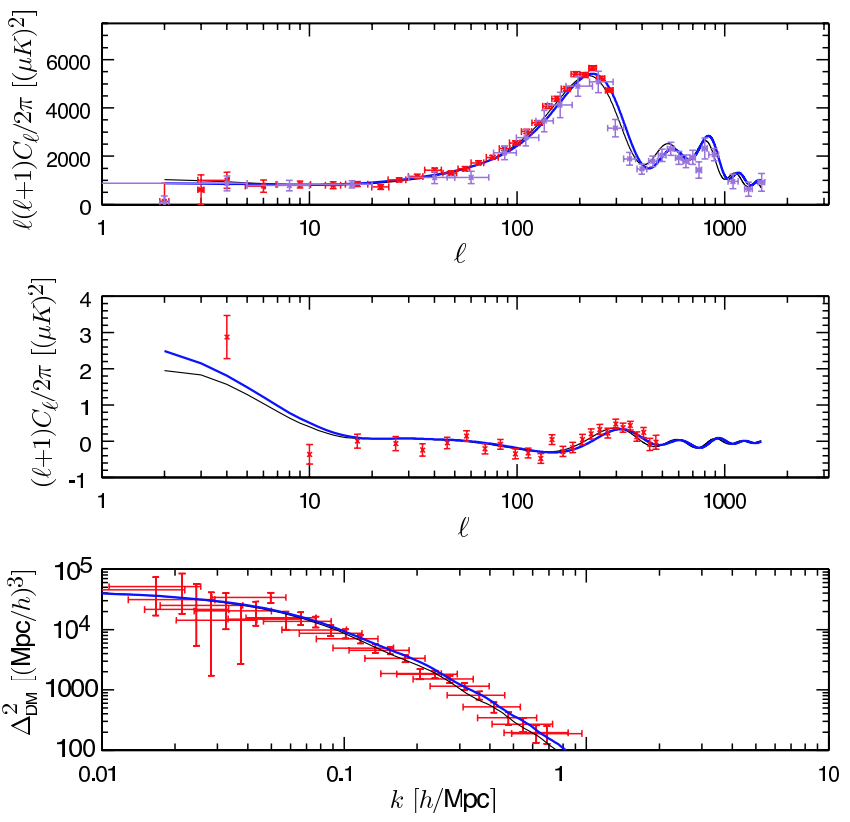

Figure 6. Angular power spectrum (top panel) and $T$ - $E$ cross-correlation (middle panel) versus the multipole $\ell$, and matter power spectrum versus the wave number $k$ (bottom panel), for the periodic equation of state (1) (blue line) and for the $\Lambda$ CDM model (black line). The top panel shows experimental data from the WMAP experiment (red) 30 and from the combination of all CMB data (purple) 31. The middle panel shows WMAP data. The data in the bottom panel are from an independent analysis of the $2 \mathrm{dF}$ survey 32 .

asł

$$
\mu \equiv m-M=5 \log _{10}\left(\frac{d_{\mathrm{L}}}{1 \mathrm{Mpc}}\right)+25 .
$$

The resolution hinges on establishing the absolute magnitude as a standard candle.

In Figure [7 we compare the luminosity distance modulus of the Supernova Search Team's gold and silver samples with the expectations of the $\Lambda$ CDM picture and the undulant universe (11). For redshifts in the range $0 \lesssim z \lesssim 2$, the models cannot now be distinguished. To quantify the range of frequency parameters $b$ that adequately reproduce the existing data, we have modified Yun Wang's supernova flux-averaging likelihood code [35, 36] to incorporate the periodic equation of state (2). In Figure 8 we show how the goodness of fit to the gold and silver sample data, measured by $\chi^{2}$, depends upon $b$. The best fit corresponds to $b=0$, the $\Lambda$ CDM solution, but a frequency parameter as large as $b=2$ is tolerated at $95 \%$ C.L. In particular, our canonical undulant model (1) delivers a $\chi^{2}$ that differs only insubstantially from the minimum value.

The equation of state (11), plotted in the upper panel of Figure [7 does increase from $w(z=0)=-1$ to $w(z=2)=-0.45$, so future high-precision measurements might make the distinction. We show the possibilities for experimental discrimination in two ways in Figure 9 The left panel shows that the difference between the implications of $\Lambda \mathrm{CDM}$ and the undulant universe becomes more pronounced with

$\ddagger$ The numerical factors are astronomical conventions. 


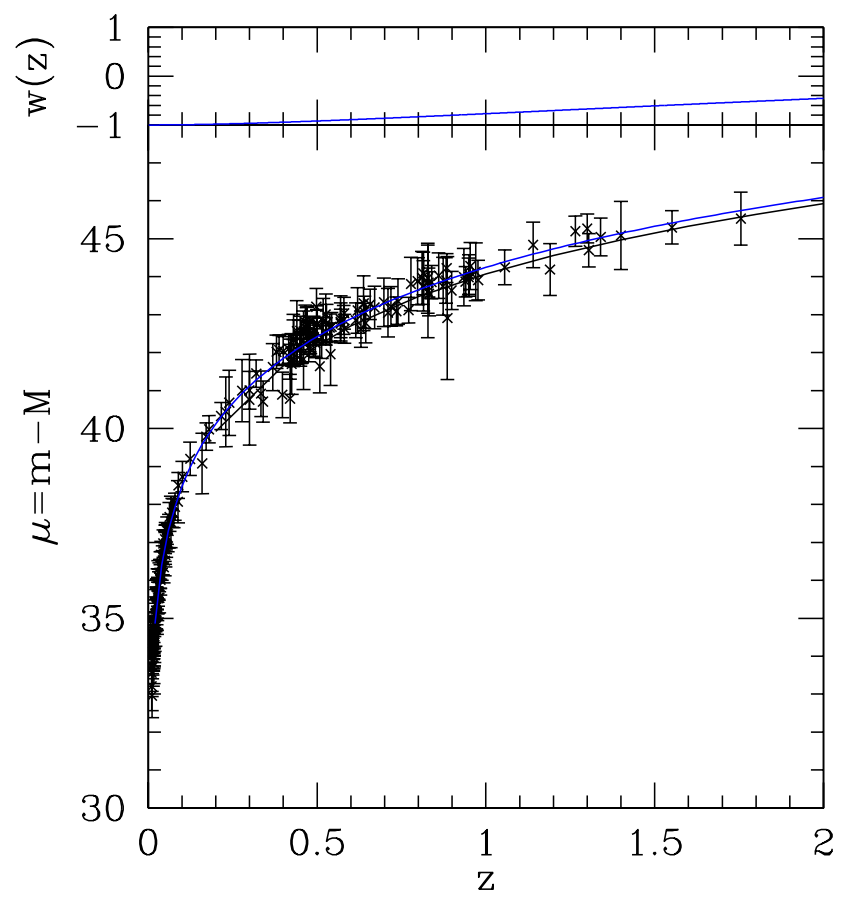

Figure 7. The distance modulus $\mu=m-M$ for the $\Lambda$ CDM model (black line) and for the undulant universe of Equation (1) (blue line), compared with the luminosity modulus-redshift data from the SN gold and silver samples [34]. The periodic equation of state is plotted in the upper panel.

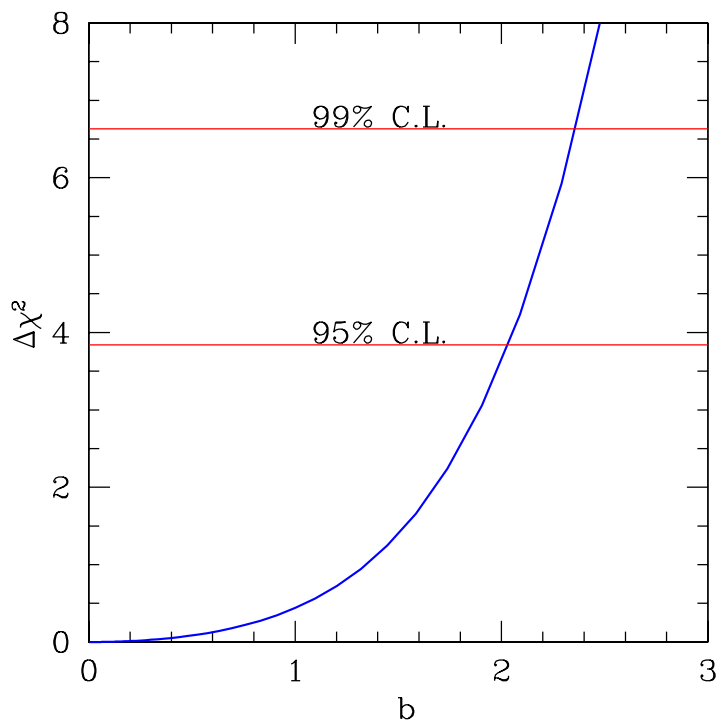

Figure 8. $\Delta \chi^{2}$ as a function of the $b$ parameter for one-parameter fits to the luminosity distance modulus. Red lines indicate the bounds at $95 \%$ and $99 \%$ C.L. 

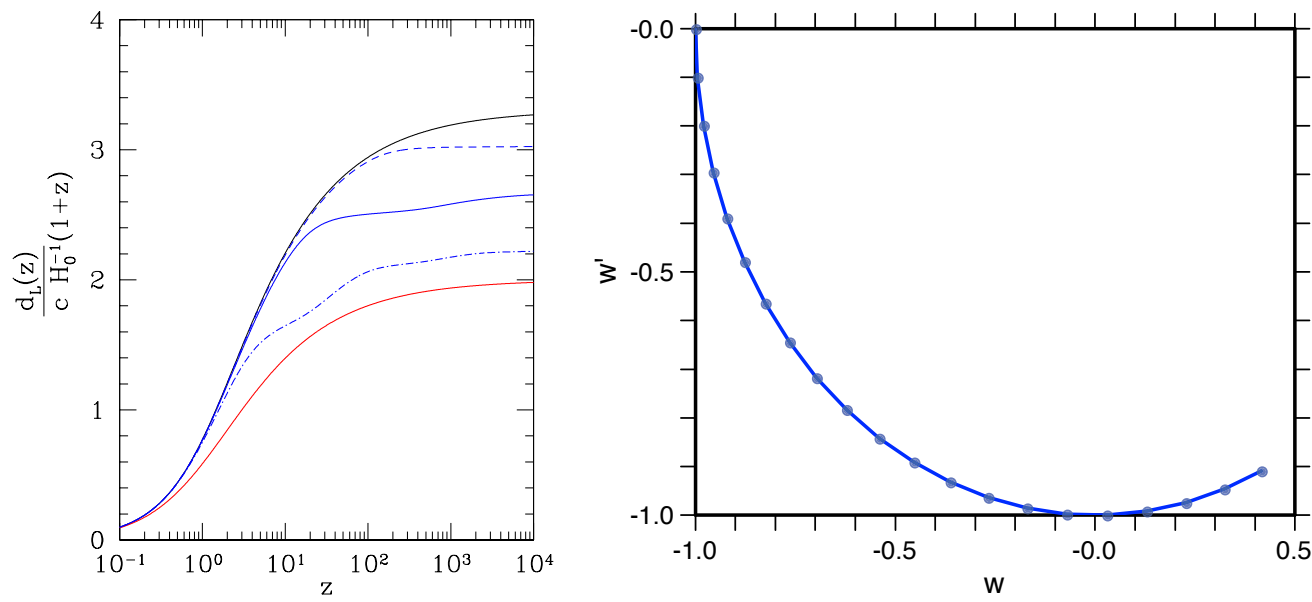

Figure 9. The left panel displays the supernova luminosity distance versus redshift for the $\Lambda \mathrm{CDM}$ (black), undulant universe of Equation (1) (solid blue), critical SCDM universe (red). Also shown are two variants of the undulant universe, defined by (2] with $b=2$ (dot-dashed blue) and $b=0.6$ (dashed blue). The right panel shows the correlation between the equation of state $w$ and its derivative $w^{\prime}=d w / d \ln a$, for the periodic equation of state [2]. The point at $(-1,0)$ corresponds to zero redshift, $\ln a=0$, and the step between points is $\Delta \ln a=-0.1 / b$.

increasing redshift. The canonical undulant universe with $b=1$ deviates appreciably from the $\Lambda \mathrm{CDM}$ for redshifts $z \gtrsim 10$; the departure occurs in the neighborhood of $z=3$ for $b=2$ and at $z \sim \mathcal{O}(100)$ for $b=0.6$. As is well known, the critical (SCDM) universe characterized by $\Omega_{m}=1$ is ruled out by observations in the neighborhood of $z=1$.

The right panel of Figure 9 shows the correlation 37] between the equation of state, $w$, and its derivative, $w^{\prime} \equiv d w / d \ln a$ for the periodic equation of state (2) near the present. The correlation is independent of the frequency parameter $b$, but the association of a particular value of $w$ with scale factor of course depends on the periodicity, as detailed in the figure caption.

Significant improvements are promised by candidates for the NASA/DOE Joint Dark Energy Mission [38]: DESTINY [39, JEDI [40, and SNAP [41, alone and in combination with cosmic microwave background results from the European Space Agency's Planck satellite [4].

\subsection{Galaxy clusters}

Measurements of the apparent redshift dependence of the baryonic mass fraction of galaxy clusters can be used to constrain the geometry of the Universe and, hence, the amount and character of dark energy. The geometry enters in the dependence of the baryonic mass fraction on the assumed angular diameter distances, $d_{\mathrm{A}}=d_{\mathrm{L}} /(1+z)^{2}$, to the clusters. The baryonic mass fraction in the largest clusters should be independent of redshift, provided that the reference cosmology used in making the baryonic mass fraction measurements matches the true cosmology of the physical Universe.

Because galaxy clusters are so large, it is plausible that they represent a fair sample of the matter in the universe, so that the relative amounts of hot gas and dark 

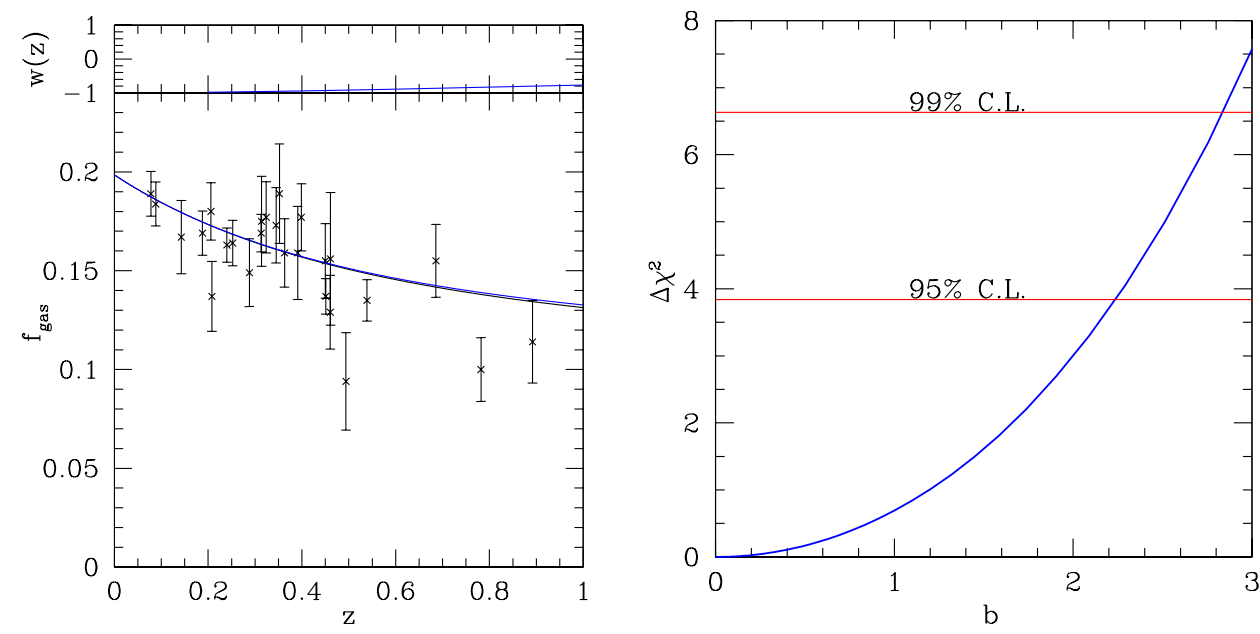

Figure 10. (Left panel) Apparent variation of the X-ray gas mass fraction (with $1 \sigma \mathrm{rms}$ errors) as function of the redshift for the reference SCDM. The expection of the $\Lambda \mathrm{CDM}$ cosmology is plotted as the black curve. The blue curve shows the prediction of the undulant universe (1). The periodic equation of state is plotted in the upper panel. (Right panel) $\Delta \chi^{2}$ results for the analysis of the X-ray mass fraction data as function of the frequency parameter $b$ of [2].

matter should be the same for every cluster. Moreover, the baryonic-to-total mass in the clusters should closely match the ratio of the cosmological parameters $\Omega_{b} / \Omega_{m}$. By measuring the $\mathrm{X}$-ray emissivity of 26 dynamically relaxed galaxy clusters in the redshift range $0.07<z<0.9$ with the Chandra X-ray Observatory 43, Allen and collaborators have determined the X-ray gas mass fraction in these systems, using the SCDM reference cosmology 44. The variation of the inferred gas fractions with redshift, shown in the left panel of Figure 10 indicates that the distance-redshift correlation of the SCDM reference cosmology does not correspond to our Universe.

To compute the expectations of cosmologies that include vacuum energy, we evaluate the gas mass fraction,

$$
f_{\text {gas }}(z)=\frac{\mathcal{B} \Omega_{\mathrm{b}}}{(1+0.19 \sqrt{h}) \Omega_{\mathrm{m}}}\left[\frac{d_{\mathrm{A}}^{\mathrm{SCDM}}(z)}{d_{\mathrm{A}}^{\mathrm{vac}}(z)}\right]^{1.5} .
$$

The bias factor $\mathcal{B}=0.824 \pm 0.089$, for which we adopt a Gaussian prior 44, 45, accounts for the relatively small amount of baryonic material expelled from galaxy clusters as they form. The $\Lambda \mathrm{CDM}$ model (black curve) and the periodic equation of state (11) (blue curve) both reproduce the data faithfully.

The gas mass fraction is sensitive not only to the amount of vacuum energy, but also to its character, because the luminosity distance defined in (9) gives some sensitivity to the equation of state through $H(z)$. The right panel of Figure 10 shows how the goodness of fit, as measured by $\chi^{2}$, depends on the frequency parameter $b$ of Equation (2). The current gas mass fraction measurements disfavor values $b \gtrsim 2.2$, so are slightly less restrictive constraint than the supernova observations. 


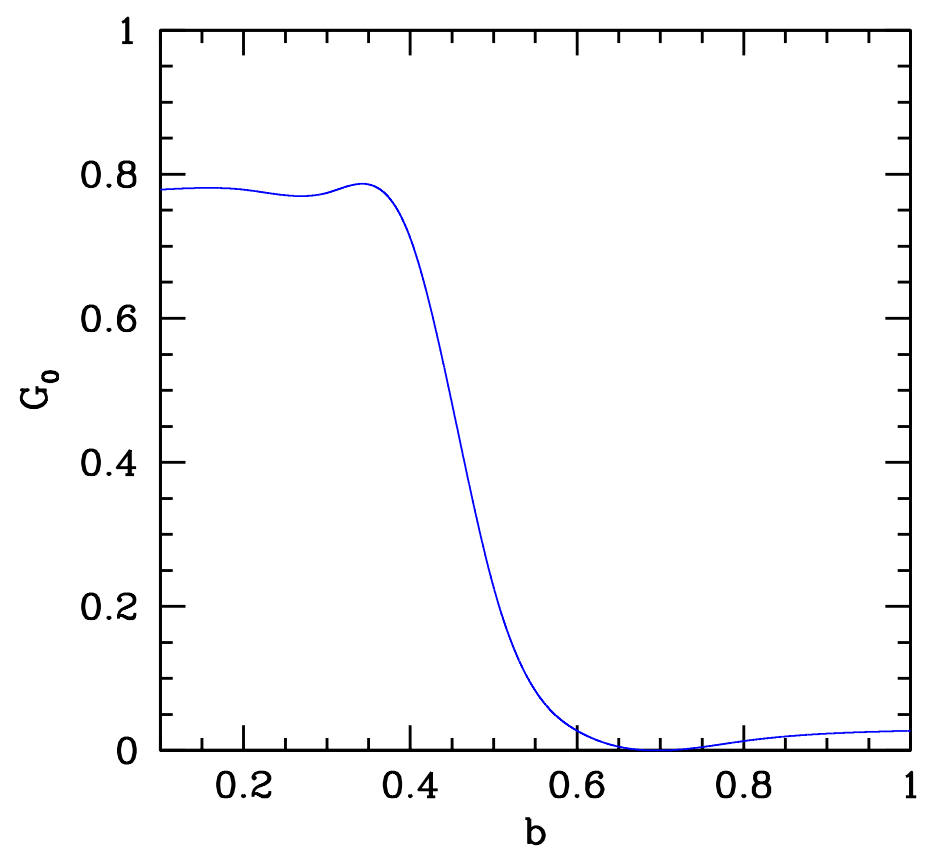

Figure 11. Linear growth factor in the present universe, $G_{0} \equiv G(a=1)$ defined through Equation 12 for an undulant universe characterized by the equation of state [2], as a function of the frequency parameter $b$.

\subsection{Structure formation}

The development of large scale structure in the universe is sensitive to the presence and character of dark energy. Vacuum energy influences the balance of attractive gravititational stability against the dynamical friction of the expansion. For an undulant universe, the equation of state of the vacuum energy affects the cosmic volume in which structures form, and rules the dynamical behavior of the dark energy.

A simple, and useful, test is provided by the linear growth factor considered by Linder and Jenkins [27. Considering linear evolution of the perturbations only, we may define the normalized growth factor

$$
G^{\prime \prime}(a)+\frac{3}{2}\left[\frac{7}{3}-\frac{w(a)}{1+X(a)}\right] \frac{G^{\prime}(a)}{a}+\frac{3}{2} \cdot \frac{1-w(a)}{1+X(a)} \frac{G(a)}{a^{2}}=0,
$$

where a prime denotes a derivative with respect to the scale factor $a$ and

$$
X(a)=\frac{\Omega_{m}}{1-\Omega_{m}} \cdot \frac{1}{g(a)},
$$

with $g(a)$ given by (6), is the ratio of matter density to dark energy density when radiation is negligible. Then, adopting the boundary condition $G(0)=1$, we may solve for the normalized growth factor $G(a)$. The resulting values at the current epoch $(a=1)$ are shown in Figure 11 for the undulant universe with over the range of frequency parameters $0 \leq b \leq 1$. 


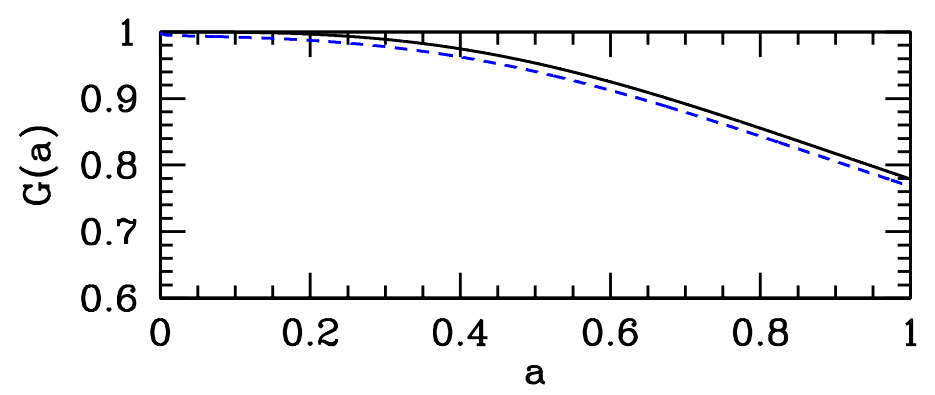

Figure 12. Dependence on scale factor $a$ of the linear growth factor $G(a)$ for the $\Lambda$ CDM cosmology (solid line) and for an undulant universe with frequency parameter $b=0.4$ (dashed line).

In the limit as $b \rightarrow 0$, the periodic equation of state (2) approaches the $\Lambda \mathrm{CDM}$ cosmology, so reproduces the canonical growth factor. The value of the growth factor in the present universe depends little on the frequency parameter for $b \lesssim 0.4$, then drops precipitously to small values. In particular, the case $b=0.4$, the consequences of which are in excellent agreement with all the other constraints we have examined, hardly differs from the $\Lambda \mathrm{CDM}$ result. Current determinations of $\sigma_{8}$ agree with the $\Lambda \mathrm{CDM}$ expectation within the uncertainties of about $20 \%$. Indeed, the $b \lessgtr 0.4$ undulant universes track the $\Lambda \mathrm{CDM}$ solution as a function of the scale factor $a$. We display the $b=0.4$ and $\Lambda \mathrm{CDM}$ solutions in Figure 12

The undulant universe characterized by frequency parameter $b=1$ fails the test of the linear growth factor, as commented by Linder [46]. While taking note of this disagreement, we believe it is prudent to note that the strategy of considering linear evolution of the perturbations, while plausible, has not been exhaustively validated. The undulant universe of Equation (1) does reproduce the matter power spectrum determined by cosmic microwave background measurements, so it is possible that a different treatment of structure formation might have a favorable outcome. Alternatively, it may be that frequency parameters $b \gtrsim 0.4$ can be brought into agreement with structure formation constraints only by delaying the emergence of the vacuum energy component or, more generally, by introducing coupling between the vacuum energy and other components, as in the slinky inflation model.

\section{Outlook}

The undulant universe offers a new response to the cosmic coincidence problem: the current state of the Universe, with $\Omega_{m} \approx \Omega_{v}$ and $w_{v} \approx-1$, has happened before and will happen again, so long as the frequency parameter $b \gtrsim 0.6$ in the undulant equation of state (2). No fine tuning is required, in the sense that $0.5 \leq \Omega_{v} \leq 0.9$ with $w_{v} \leq-0.7$ occurs with $\sim 9 \%$ probability for $10^{-7} \leq a \leq 10^{+7}$ for the prototype undulant universe with $b=1$. The corresponding number is $4.5 \%$ for the case $b=0.4$. [See the details in Table 1]

We find that periodic equations of state (2) reproduce the correct power spectrum 
and CMB temperature asymmetries, so long as the frequency parameter $b \lessgtr 2$. Moreover, such models satisfy constraints on the matter density at the time of big bang nucleosynthesis, except for frequency parameters in the neighborhood of $b \approx \frac{1}{4}$. The undulant cosmologies fit very well late-time geometry probes such as supernova luminosity distances; at 95\% C.L., frequency parameters as large as $b=2$ are acceptable. The one delicate issue is structure formation as embodied in the growth factor. A linear treatment of the evolution of density perturbations restricts the frequency parameter to $b \lessgtr 0.4$. The case of $b=0.4$ is in comfortable agreement with all observations, but entails only the present matter-dark-energy coincidence since the radiation-dominated era ended.

We have postulated the periodic equation of state, not derived it from a dynamical principle, in the interest of exploring alternative implications of the discovery of the accelerating universe. The more ambitious program of slinky inflation 16 gives an explicit construction of a periodic equation of state from a potential that governs the behavior of a scalar field [47, 48, 49].

On the observational front, it is of clear interest to pin down the vacuum-energy equation of state $w(z)$ and to seek evidence that it varies with time, for redshifts $z \approx 1$ [50, 51]. This observational challenge is an imperative for quintessence models in general, for the $\Lambda \mathrm{CDM}$ picture, and for the undulant universe and its extension, slinky inflation. The observational survival of periodic equations of state highlights the fact that, at present, we have telling observations at only a few epochs in the history of the universe. It is of clear interest to devise new observational tests that will explore new ranges in redshift, making new strata of the fossil record available for our scrutiny. The wide excursions in the deceleration parameter at different epochs, shown in Figure 5 offer encouragement for probing deeper in redshift than is possible with supernovae.

While no finite set of astronomical measurements made over a finite time will ever allow us to determine the ultimate fate of our Universe [52], we can hope to look some distance into the future. Wang \& collaborators have quantified [53] the limited reach of reliable extrapolations in the framework of the simplest doomsday model, in which the universe collapses swiftly, once it ceases to expand. They reckon the collapse time $t_{\text {collapse }} \gtrsim 42(24)$ Gyr from today at $68 \%$ (95\%) C.L. An interesting parameter in the undulant universe is the moment that marks the onset of the next period of deceleration, which we characterize as the moment at which the deceleration parameter next exceeds the value that obtains in a matter-critical universe, $q_{\mathrm{SCDM}}=\frac{1}{2}$. That circumstance occurs at $(11,23,39,153) \mathrm{Gyr}$ in the future for frequency parameters $b=(3,2,1,0.4)$. The cases with $b \lesssim 2$ all respect the doomsday bounds of Ref. [53].

The undulant universe explored here serves as a reminder that the range of possible destinies for the Universe, even in the near term, is very broad indeed. The universe need not necessarily evolve toward the cataclysm of terminal inflation or recollapse, but might steer a middle course not so different, on average, from a critical universe dominated by matter. The main lesson of the undulant universe is that it is premature to anoint the $\Lambda \mathrm{CDM}$ model as the sole candidate for the new standard cosmology.

\section{Acknowledgments}

Fermilab is operated by Universities Research Association Inc. under Contract No. DEAC02-76CH03000 with the U.S. Department of Energy. It is a pleasure to thank Steve 
Allen, Eric Linder, Joe Lykken and Matias Zaldarriaga for helpful communications.

\section{References}

[1] Peebles PJE and Ratra B, The cosmological constant and dark energy, 2003 Rev. Mod. Phys. 75559 [astro-ph/0207347]

[2] Freedman WL and Turner MS, Measuring and understanding the universe, 2003 Rev. Mod. Phys. 751433 [astro-ph/0308418]

[3] Trodden M and Carroll SM, Introduction to Cosmology, in Particle Physics and Cosmology: The Quest for Physics beyond the Standard Model(s): TASI 2002, Haber HE and Nelson AE, Eds. (World Scientific, Singapore, 2004), pp. 703-793 [astro-ph/0401547]

[4] Riess AG et al [Supernova Search Team], Observational Evidence from Supernovae for an Accelerating Universe and a Cosmological Constant, 1998 Astron. J. 1161009 [astro$\mathrm{ph} / 9805201]$

[5] Perlmutter S et al [Supernova Cosmology Project], Measurements of $\Omega$ and $\Lambda$ from 42 HighRedshift Supernovae, 1999 Astrophys. J. 517565 [astro-ph/9812133]

[6] Bennett CL et al, First Year Wilkinson Microwave Anisotropy Probe (WMAP) Observations: Preliminary Maps and Basic Results, 2003 Astrophys. J. Suppl. 148 1 [astro-ph/0302207]. A summary table of cosmological parameters is available at http://lambda.gsfc.nasa.gov/product/map/wmap_parameters.cfm

[7] Cole S et al [The Two-Degree Field Galaxy Redshift Survey Collaboration], The 2dF Galaxy Redshift Survey: Power-spectrum analysis of the final dataset and cosmological implications [astro-ph/0501174]. The 2dF survey obtained spectra for 245591 objects, mainly galaxies; see http://www.mso.anu.edu.au/2dFGRS/

[8] Tegmark $\mathrm{M}$ et al [SDSS Collaboration], Cosmological parameters from SDSS and WMAP, 2004 Phys. Rev. D 69103501 [astro-ph/0310723]. The Sloan Digital Sky Survey, http://www.sdss.org measures distances to more than a million galaxies and quasars.

[9] Bridle SL, Lahav O, Ostriker JP and Steinhardt PJ, Precision Cosmology? Not Just Yet, 2003 Science 2991532 [astro-ph/0303180]

[10] Krauss LM and Turner MS, The cosmological constant is back, 1995 Gen. Rel. Grav. 271137 [astro-ph/9504003]

[11] Caldwell RR, Dave R and Steinhardt PJ, Cosmological Imprint of an Energy Component with General Equation-of-State, 1998 Phys. Rev. Lett. 801582 [astro-ph/9708069]

[12] Wang LM, Caldwell RR, Ostriker JP and Steinhardt PJ, Cosmic Concordance and Quintessence, 2000 Astrophys. J. 53017 [astro-ph/9901388].

[13] Susskind L, The anthropic landscape of string theory [hep-th/0302219]

[14] Steinhardt PJ and Turok N, A cyclic model of the universe, 2002 Science 2961436

[15] Barenboim G, Mena O and Quigg C, Undulant universe: Expansion with alternating eras of acceleration and deceleration, 2005 Phys. Rev. D 71063533 [astro-ph/0412010]

[16] Barenboim G and Lykken J, Slinky inflation [astro-ph/0504090]

[17] Feng B, Li M, Piao YS and Zhang X, Oscillating quintom and the recurrent universe, [astro$\mathrm{ph} / 0407432]$

[18] Guo ZK, Piao YS, Zhang XM and Zhang YZ, Cosmological evolution of a quintom model of dark energy, 2005 Phys. Lett. B 608177 [astro-ph/0410654]

[19] Xia JQ, Feng B and Zhang XM, Constraints on Oscillating Quintom from Supernova, Microwave Background and Galaxy Clustering, [astro-ph/0411501]

[20] Wei H, Cai RG and Zeng DF, Hessence: A new view of quintom dark energy, [hep-th/0501160]

[21] Lazkoz R, Nesseris S and Perivolaropoulos L, Evidence for Cosmological Oscillations in the Gold SnIa Dataset, [astro-ph/0503230]

[22] Eidelman S et al [Particle Data Group], Review of particle physics, 2004 Phys. Lett. B 5921

[23] Dodelson S, Kaplinghat M, and Stewart E, Tracking oscillating energy, 2000 Phys. Rev. Lett. 855276 [astro-ph/0002360]

[24] Griest K, Toward a possible solution to the cosmic coincidence problem, 2002 Phys. Rev. D 66 123501 [astro-ph/0202052]

[25] Sahni V and Wang LM, A New cosmological model of quintessence and dark matter, 2000 Phys. Rev. D 62, 103517 [astro-ph/9910097]

[26] Ferrer F and Rasanen S, Dark energy and decompactification in string gas cosmology, [hepth/0509225]

[27] Linder EV and Jenkins A, Cosmic Structure and Dark Energy, 2003 Mon. Not. Roy. Astron. Soc. 346573 [astro-ph/0305286] 
[28] Bean R, Hansen SH and Melchiorri A, Early universe constraints on dark energy, 2001 Phys. Rev. D 64103508 [arXiv:astro-ph/0104162]

[29] Seljak U and Zaldarriaga M, A Line of Sight Approach to Cosmic Microwave Background Anisotropies, 1996 Astrophys. J. 469437 [astro-ph/9603033]

The most recent version of the CMBFAST code is at http://www.cmbfast.org/

[30] WMAP power spectrum data can be found at http://lambda.gsfc.nasa.gov/product/map/

[31] Wang X, Tegmark M, Jain B and Zaldarriaga M, The last stand before MAP: cosmological parameters from lensing, CMB and galaxy clustering, 2003 Phys. Rev. D 68123001 [astro$\mathrm{ph} / 0212417]$

[32] Tegmark M, Hamilton AJS and Xu Y, The power spectrum of galaxies in the 2dF 100k redshift survey, 2002 Mon. Not. Roy. Astron. Soc. 335887 [astro-ph/0111575]

[33] de Oliveira-Costa A, Tegmark M, O'Dell C, Keating B, Timbie P, Efstathiou G, and Smoot G, The large-scale polarization of the microwave background and foreground, 2003 Phys. Rev. D 68083003 [arXiv:astro-ph/0212419]

[34] Riess AG et al [Supernova Search Team], Type Ia Supernova Discoveries at $z>1$ from the Hubble Space Telescope: Evidence for Past Deceleration and Constraints on Dark Energy Evolution, 2004 Astrophys. J. 607665 [astro-ph/0402512]

[35] Wang Y, Flux-averaging Analysis of Type Ia Supernova Data, 2000 Astrophys. J. 536531 [astro-ph/9907405]; Wang Y and Mukherjee P, Model-Independent Constraints on Dark Energy Density from Flux-averaging Analysis of Type Ia Supernova Data, 2004 Astrophys. J. 606654 [astro-ph/0312192]; Wang Y and Tegmark M, New dark energy constraints from supernovae, microwave background and galaxy clustering, 2004 Phys. Rev. Lett. 92241302 [astro-ph/0403292]

[36] The most recent version of the code is at http://www.nhn.ou.edu/ ${ }^{\sim}$ wang/SNcode/index.html

[37] Linder EV, Exploring the expansion history of the universe, 2003 Phys. Rev. Lett. 90091301 [arXiv:astro-ph/0208512].

[38] JDEM: NASA/DOE Joint Dark Energy Mission,http://spacescience.nasa.gov/admin/divisions/sz/SEUS0310/JDEM_Roadmap.pdf

[39] DESTINY: Dark Energy Space Telescope, http://destiny.asu.edu/

[40] JEDI: Joint Efficient Dark-Energy Investigation, http://jedi.nhn.ou.edu/

[41] SNAP: Supernova / Acceleration Probe, http://snap.lbl.gov/

[42] The Planck Satellite, http://www.rssd.esa.int/index.php?project=PLANCK

[43] The Chandra X-Ray Observatory, http://chandra.nasa.gov

[44] Allen SW, Schmidt RW, Ebeling H, Fabian HC and van Speybroeck L, Constraints on dark energy from Chandra observations of the largest relaxed galaxy clusters, 2004 Mon. Not. Roy. Astron. Soc. 353457 [astro-ph/0405340]

[45] Rapetti D, Allen SW and Weller J, Constraining Dark Energy with X-ray Galaxy Clusters, Supernovae and the Cosmic Microwave Background, 2005 Mon. Not. Roy. Astron. Soc. 360 555 [astro-ph/0409574]

[46] Linder EV, Cosmic growth history and expansion history, 2005 Phys. Rev. D 72043529 [arXiv:astro-ph/0507263]

[47] Nakamura T and Chiba T, Determining the equation of state of the expanding universe: Inverse problem in cosmology, 1999 Mon. Not. Roy. Astron. Soc. 306696 [astro-ph/9810447]

[48] Gerke BF and Efstathiou G, Probing quintessence: Reconstruction and parameter estimation from supernovae, 2002 Mon. Not. Roy. Astron. Soc. 33533 [astro-ph/0201336]

[49] Guo ZK, Ohta N and Zhang YZ, Parametrization of quintessence and its potential, 2005 Phys. Rev. D 72, 023504 [astro-ph/0505253].

[50] Alam U, Sahni V, Saini TD and Starobinsky AA, Is there Supernova Evidence for Dark Energy Metamorphosis? 2004 Mon. Not. Roy. Astron. Soc. 354275 [astro-ph/0311364]

[51] Jassal HK, Bagla JS and Padmanabhan T, Observational constraints on low redshift evolution of dark energy: How consistent are different observations?, [astro-ph/0506748].

[52] Krauss LM and Turner MS, Geometry and destiny, 1999 Gen. Rel. Grav. 311453 [astro$\mathrm{ph} / 9904020]$

[53] Wang Y, Kratochvil JM, Linde A and Shmakova M, Current Observational Constraints on Cosmic Doomsday, 2004 JCAP 0412006 [astro-ph/0409264] 